\title{
$A b$ initio energies of nonconducting crystals by systematic fragmentation
}

\author{
Heather M. Netzloff ${ }^{\text {a) }}$ and Michael A. Collins ${ }^{\text {b) }}$ \\ Research School of Chemistry, Australian National University, Canberra, \\ Australian Capital Territory 0200, Australia
}

(Received 29 May 2007; accepted 11 July 2007; published online 5 October 2007)

\begin{abstract}
A systematic method for approximating the ab initio electronic energy of molecules from the energies of molecular fragments has been adapted to estimate the total electronic energy of crystal lattices. The fragmentation method can be employed with any ab initio electronic structure method and allows optimization of the crystal structure based on $a b$ initio gradients. The method is demonstrated on $\mathrm{SiO}_{2}$ polymorphs using the Hartree-Fock approximation, second order Möller-Plesset perturbation theory, and the quadratic configuration interaction method with single and double excitations and triple excitations added perturbatively . (c) 2007 American Institute of Physics. [DOI: 10.1063/1.2768534]
\end{abstract}

\section{INTRODUCTION}

A primary aim of theoretical and computational chemistry is to accurately calculate properties of chemical systems. These properties include energetics and thermochemical properties, structure, and reaction dynamics. Ab initio quantum chemistry provides the means to do this for small- to moderate-sized molecules. A hierarchy of techniques exists, ranging from the fundamental Hartree-Fock (HF) approach and density functional theory (DFT) to methods that include some degree of electron correlation, such as second order Möller-Plesset perturbation theory (MP2) and the coupledcluster approximation with single and double excitations (CCSD). The main limitation in the progression up the scale is computational cost (the "scaling problem"): as the size of the system (number of electrons, $n$ ) increases, so does the computational time, depending on the level of theory. For example, MP2 scales as $\mathrm{O}\left(n^{5}\right)$, while CCSD scales as $\mathrm{O}\left(n^{6}\right)$.

The study of solid-state materials, especially with the use of periodic, high-level calculations, has followed its molecular counterpart in making significant advances over the years. Since solid-state systems, including crystals, are much larger than molecules, calculation methods were originally limited to model potential schemes or semiclassical (atomistic) studies. At present, the most widely used electronic structure method for periodic systems is DFT, despite concerns that the method has been proven many times to be unpredictable and difficult to systematically improve, does not describe dispersive forces, ${ }^{1}$ and underestimates transition state energies and band gaps. An accurate and reliable description of the effect of electron correlation on crystal properties is needed.

Several programs and methods do exist for periodic solid-state electronic structure calculations. The CRYSTAL program $^{2}$ calculates the energy and gradients, optimizes geometries, and obtains many structural features with HF and DFT at a periodic $a b$ initio level. A derivative of CRYSTAL,

\footnotetext{
a)Electronic mail: heather@si.fi.ameslab.gov

${ }^{b)}$ Electronic mail: collins@rsc.anu.edu.au
}

CRYSCOR, ${ }^{3}$ includes periodic local correlation at the MP2 level of theory. Susceria and co-workers have generalized their molecular Laplace MP2 method $^{4,5}$ for periodic systems. ${ }^{6}$ Quantum Monte Carlo methods are also increasingly being applied to solids. ${ }^{7}$ The main aim of this paper is to propose a new, relatively simple systematic method to investigate crystals with high levels of $a b$ initio electronic structure theory.

For molecules, a recent approach to the scaling problem of quantum electronic structure calculations is fragmentation. The general idea of breaking a molecule into fragments, evaluating molecular orbitals (or electron density or the density matrix) for these fragments, and then combining results to establish quantities for the whole molecule has existed for many years (see, for example, Refs. 8-11). Zhang and Zhang ${ }^{12}$ developed a simple idea for estimating the interaction energy between two large molecules from the interaction of fragments of the two molecules (fragments are overlapping segments) with good results.

In recent years, a systematic fragmentation method for large molecules, based on the work by Zhang and Zhang, has been developed. ${ }^{13,14}$ A molecule is decomposed into a set of small fragments; the energy of each fragment is calculated, and the total energy of the molecule is then approximated as a linear sum of the fragment energies. A systematic hierarchy of fragmentation approximations was developed and automated to provide a sequence of increasingly reliable estimates for the total energy of the molecule. A major feature of this approach is that a very large molecule can be broken into a number of small fragments, making high-level $a b$ initio calculations feasible. Linear scaling in computation time is achieved, and the procedure is highly parallel, as all fragment energies can be evaluated on separate processors. This approach considers the interaction of the molecular fragments in terms of "bonded" interactions. In addition, relatively short range nonbonded interactions are treated with $a b$ initio calculations involving small molecular fragments, and long range nonbonded interactions are treated with an electrostatics approximation, ensuring that computational time for nonbonded interactions also scales linearly with the size 
of the molecule. This approach has been shown to accurately estimate the energy of nonaromatic molecules. Not surprisingly, however, the energy of an aromatic molecule such as benzene cannot be accurately estimated from the energies of fragments of the ring.

It is in the spirit of this fragmentation for large nonaromatic molecules that we propose a new method for treating nonconducting crystals by systematic fragmentation of the bonding structure. The molecular fragmentation process has been modified to account for periodicity. This first paper presents the fragmentation method for crystals and illustrates the resultant energy calculations for four systematic levels of fragmentation of eight structural phases of $\mathrm{SiO}_{2}$. A systematic treatment of the nonbonded interactions which contribute to the crystal energy is also presented. In this first paper, energy gradients for the bonded interactions are described and geometry optimizations based on these interactions only are presented. The modification necessary to calculate crystal structures at constant pressure is also described and illustrated with calculations. Evaluation of second derivatives of the energy can also be achieved efficiently in this approach, leading to the calculation of phonon frequencies. However, the calculation of phonon frequencies is not included here.

The paper is laid out as follows: first, a short description of the fragmentation method for large molecules will be presented, followed by modifications for crystal periodicity. The total crystal energy and gradient, derived from the "fragmented" crystal, will be presented, as well as optimization within this scheme. Silicon dioxide $\left(\mathrm{SiO}_{2}\right)$, silica, and its series of polymorphs have been chosen as the initial test case. $\mathrm{SiO}_{2}$ has widespread occurrence in many natural phases and has technological importance in catalysis, microelectronics, and optical fiber devices. Static calculations of these structures are important to correctly predict the subtle structural differences between phases, as well as the relative stability of the polymorphs as a function of temperature and pressure. The results of energy calculations for $\mathrm{SiO}_{2}$ polymorphs will be presented and geometry optimizations will be demonstrated. Some concluding remarks complete the paper.

\section{FRAGMENTATION}

A large molecule or crystal is broken up into small molecular fragments in order to estimate the total energy of the molecule or crystal from the energies of the small molecular fragments. A detailed description of the fragmentation method for molecules has been given in two previous papers, ${ }^{3,14}$ so only a simple review is presented to introduce the ideas that have been employed to fragment a crystal structure.

\section{A. Background}

A molecule or crystal is viewed as a collection of chemically bonded atoms, collected into functional groups, defined in the usual way. Hydrogen atoms belong to the functional group of the heavy atom to which they are bonded. Atoms connected by double or triple bonds belong to the same functional group. Ethanol, for example, contains three functional groups $\left(\mathrm{CH}_{3}, \mathrm{CH}_{2}\right.$, and $\left.\mathrm{OH}\right)$, while ethanal (acetaldehyde) contains just two functional groups $\left(\mathrm{CH}_{3}\right.$ and $\left.\mathrm{CHO}\right)$. These groups are therefore connected by single bonds, and a molecule or crystal is broken into fragments by breaking these single bonds between groups.

When a bond between functional groups in a molecule or crystal is broken, the bonding is restored by appending hydrogen atoms to each group. Thus, breaking all the intergroup bonds in ethanol produces three isolated fragment molecules, $\mathrm{CH}_{4}, \mathrm{CH}_{4}$, and $\mathrm{H}_{2} \mathrm{O}$. Similarly, if the bonds are broken which connect a sequence of $\mathrm{SiOSi}$ atoms to a crystal of $\mathrm{SiO}_{2}$, the molecular fragment $\left(\mathrm{SiH}_{3}\right) \mathrm{O}\left(\mathrm{SiH}_{3}\right)$ is produced.

It is important to emphasize that in all other respects, the shape of a fragment molecule is identical to the shape it had in the parent molecule or crystal.

The rationale for breaking a molecule (or crystal) into fragments is best understood using a simple example: a chain molecule $M$ containing $K$ functional groups:

$$
M=G_{1} G_{2} G_{3}, \ldots, G_{K} .
$$

$M$ can be broken between groups $n-1$ and $n$ by stretching the $G_{n-1} G_{n}$ single bond to infinity and replacing the $G_{n-1} G_{n}$ bond by hydrogen "caps," $\mathrm{H}^{(n-1)}$ bonded to $G_{n-1}$, and $\mathrm{H}^{(n)}$ bonded to $G_{n}$ :

$$
M \rightarrow M_{1}+M_{2},
$$

where

$$
\begin{aligned}
& M_{1}=G_{1} G_{2} G_{3}, \ldots, G_{n-1} \mathrm{H}^{(n-1)}, \\
& M_{2}=\mathrm{H}^{(n)} G_{n} G_{n+1}, \ldots, G_{K} .
\end{aligned}
$$

The geometry of fragments $M_{1}$ and $M_{2}$ is preserved except for the additional hydrogen caps. As pioneered in the ONIOM method, ${ }^{15}$ the caps are located along the "missing" bond vector at an appropriate distance for the particular $G \mathrm{H}$ bond (proportional to the atomic radii).

The total energy for $M$ can be written exactly as

$$
E(M)=E\left(M_{1}\right)+E\left(M_{2}\right)+d E_{1},
$$

where $d E_{1}$ represents the net energy change due to breaking bonds and "adding" hydrogen caps. Bonds can be broken at any place. Thus, the process can be repeated, first restoring the $G_{n-1} G_{n}$ bond and then breaking $M$ at some other place, for example, stretching the $G_{i-1} G_{i}$ single bond to infinity. This yields

$$
E(M)=E\left(M_{3}\right)+E\left(M_{4}\right)+d E_{2},
$$

where

$$
\begin{aligned}
& M_{3}=G_{1} G_{2} G_{3}, \ldots, G_{i-1} \mathrm{H}^{(i-1)}, \\
& M_{4}=\mathrm{H}^{(i)} G_{i} G_{i+1}, \ldots, G_{K} .
\end{aligned}
$$

Suppose $M$ is now broken in both places simultaneously,

$$
\begin{aligned}
M & \rightarrow G_{1} G_{2} G_{3}, \ldots, G_{n-1} \mathrm{H}^{(n-1)} \\
& +\mathrm{H}^{(n)} G_{n} G_{n+1}, \ldots, G_{i-1} \mathrm{H}^{(i-1)}+\mathrm{H}^{(i)} G_{i} G_{i+1}, \ldots, G_{K} .
\end{aligned}
$$

This gives 


$$
E(M)=E\left(M_{1}\right)+E\left(M_{5}\right)+E\left(M_{4}\right)+d E_{3},
$$

where

$$
M_{5}=\mathrm{H}^{(n)} G_{n} G_{n+1}, \ldots, G_{i-1} \mathrm{H}^{(i-1)} .
$$

The reason for considering breaking bonds in this way is based on the following supposition: If the $G_{n-1} G_{n}$ bond is spatially far from the $G_{i-1} G_{i}$ bond, then the change in energy from the simultaneous breaks will be approximately equal to the sum of the energy changes from separate fragmentations.

$$
d E_{3} \approx d E_{1}+d E_{2} .
$$

The more separated the bonds, the more accurate the equality. Taking into account the above definitions, Eq. (2.12) becomes

$$
\begin{gathered}
E(M)-E\left(M_{1}\right)-E\left(M_{5}\right)-E\left(M_{4}\right) \approx E(M)-E\left(M_{1}\right) \\
-E\left(M_{2}\right)+E(M)-E\left(M_{3}\right)-E\left(M_{4}\right),
\end{gathered}
$$

or,

$$
E(M) \approx E\left(M_{2}\right)+E\left(M_{3}\right)-E\left(M_{5}\right)
$$

In this way, $M_{5}$ is the overlapping segment common to both $M_{2}$ and $M_{3}$.

The fragmentation products are determined by the location of the two bonds broken (one at a time or both together). The systematic levels of fragmentation are defined in the following way.

Level 1. The two bonds are separated by one functional group (fragmentation will account for all $\alpha$ substituent effects).

Level 2. The two bonds are separated by two functional groups (fragmentation will account for all $\alpha$ and $\beta$ substituent effects).

Level 3. The two bonds are separated by three functional groups (fragmentation will account for all $\alpha, \beta$, and $\gamma$ substituent effects).

This scheme can, of course, be continued with higher and higher levels of fragmentation.

A single application of the fragmentation scheme (for the desired level) breaks a molecule into, for example, three fragments. Each fragment has an associated sign $( \pm 1)$. Each of these fragments can then be broken into smaller fragments following the same rules. Thus, fragmentation continues at level $k$ until, with each new generation of fragments produced, the rule for bond breaking in level $k$ cannot be applied. Many of the fragments generated will cancel (fragments are generated twice and in one instance with $a+1$ sign while the other has $a-1$ sign); the survivors represent the fragmentation of $M$ at level $k$. In general, a molecule $M$ is represented by a sum and difference of $N_{\text {frag }}$ molecular fragments

$$
M \rightarrow \sum_{n=1}^{N_{\text {frag }}} c_{n} F_{n},
$$

where $F_{n}$ represents a molecular fragment and $c_{n}$ are the associated coefficients (often \pm 1 ). The final estimate of the molecular energy due to bonding between groups is

$$
E^{\mathrm{b}}(M)=\sum_{n=1}^{N_{\text {frag }}} c_{n} E\left(F_{n}\right) .
$$

The number of fragments is linearly proportional to the number of functional groups in the molecule. Hence, the $a b$ initio computational time to evaluate the right-hand side of Eq. (2.15) increases only linearly with the size of the molecule.

Gradients and higher derivatives of the energy with respect to nuclear positions can be derived in terms of the corresponding derivatives of fragment energies. Any molecular property that can be expressed as a derivative of the Hamiltonian can be expressed as a sum of the corresponding property over the fragments.

A simple example serves to illustrate the method: consider the fragmentation of $n$-pentane at level 2 . The molecule $n$-pentane can be represented as a chain of five functional groups, denoted 12345. Bonds can only be broken (one at a time or simultaneously) if they are separated by two functional groups.

$$
\begin{aligned}
12345 & \rightarrow(1+2345)+(123+45)-(1+23+45) \\
& \rightarrow 123-23+2345 \rightarrow 123-23+(2+345) \\
& +(234+5)-(2+34+5) \rightarrow 123+234 \\
& +345-23-34 .
\end{aligned}
$$

This means that the energy of any configuration of an $n$-pentane molecule should be estimated by the energy of three configurations of propane less the energy of two configurations of ethane.

The rules that define the hierarchy of fragmentation levels have been incorporated into a computer program that can fragment any molecule (not simply linear chains). Two previous papers ${ }^{13,14}$ have demonstrated that by level 3 , the total energy of a large number of typical organic molecules (with general formula $\mathrm{C}_{7-30} \mathrm{~N}_{0-7} \mathrm{O}_{0-7} \mathrm{~F}_{0-3} \mathrm{H}_{1-80}$ ) is calculated to within a few millihartrees. For a large molecule, functional groups that are well separated in terms of bonded connectivity may still be close together in space as molecules can fold in many ways. There is therefore a contribution to the total electronic energy of the molecule as a result of these "nonbonded" interactions. In previous papers, it has been shown that this nonbonded energy can be estimated from the interactions of small molecular fragments. Including this nonbonding energy with the bonding energy of Eq. (2.15) provides an estimate of the relative energies of isomers to within a few $\mathrm{kJ} \mathrm{mol}^{-1}$ for the organic molecules in Ref. 14. Hence, for moderate-sized organic molecules, the systematic fragmentation method (including nonbonded interactions) can estimate relative molecular energies to chemical accuracy.

\section{B. Crystal fragmentation}

To see how the systematic fragmentation methods can be applied to crystals, again consider a simple example, a onedimensional (1D) lattice (similar to a chain molecule). Suppose that there are three functional groups in the unit cell of this 1D lattice, labeled A, B, and C. By analogy with Eq. (2.16), fragmentation of such a 1D lattice at level 2 gives 


$$
\begin{aligned}
\cdots & A_{n-1} B_{n-1} C_{n-1} A_{n} B_{n} C_{n} A_{n+1} B_{n+1} C_{n+1} \cdots \rightarrow \cdots \\
& +A_{n-1} B_{n-1} C_{n-1}+B_{n-1} C_{n-1} A_{n}+C_{n-1} A_{n} B_{n}+A_{n} B_{n} C_{n} \\
& +B_{n} C_{n} A_{n+1}+C_{n} A_{n+1} B_{n+1}+\cdots \cdots-A_{n-1} B_{n-1} \\
& -B_{n-1} C_{n-1}-C_{n-1} A_{n}-A_{n} B_{n}-B_{n} C_{n}-C_{n} A_{n+1}-\cdots \\
& \rightarrow \sum_{n=-\infty}^{\infty}\left[B_{n-1} C_{n-1} A_{n}+C_{n-1} A_{n} B_{n}+A_{n} B_{n} C_{n}\right. \\
& \left.-B_{n-1} C_{n-1}-C_{n-1} A_{n}-A_{n} B_{n}\right] .
\end{aligned}
$$

Thus, at level 2 the energy of this lattice due to bonding between functional groups is given by

$$
\begin{aligned}
E_{1 \mathrm{D}}^{(2)}= & \sum_{n=-\infty}^{\infty}\left[E\left(B_{n-1} C_{n-1} A_{n}\right)+E\left(C_{n-1} A_{n} B_{n}\right)+E\left(A_{n} B_{n} C_{n}\right)\right. \\
& \left.-E\left(B_{n-1} C_{n-1}\right)-E\left(C_{n-1} A_{n}\right)-E\left(A_{n} B_{n}\right)\right] .
\end{aligned}
$$

Similarly, the level 1 and level 3 fragmentations of this 1D lattice would be, respectively,

$$
\begin{aligned}
E_{1 \mathrm{D}}^{(1)}= & \sum_{n=-\infty}^{\infty}\left[E\left(C_{n-1} A_{n}\right)+E\left(A_{n} B_{n}\right)+E\left(B_{n} C_{n}\right)-E\left(C_{n-1}\right)\right. \\
& \left.-E\left(A_{n}\right)-E\left(B_{n}\right)\right],
\end{aligned}
$$

and

$$
\begin{aligned}
E_{1 \mathrm{D}}^{(3)}= & \sum_{n=-\infty}^{\infty}\left[E\left(A_{n-1} B_{n-1} C_{n-1} A_{n}\right)+E\left(B_{n-1} C_{n-1} A_{n} B_{n}\right)\right. \\
& +E\left(C_{n-1} A_{n} B_{n} C_{n}\right)-E\left(A_{n-1} B_{n-1} C_{n-1}\right) \\
& \left.-E\left(B_{n-1} C_{n-1} A_{n}\right)-E\left(C_{n-1} A_{n} B_{n}\right)\right] .
\end{aligned}
$$

Note that the summands in Eqs. (2.18)-(2.20) represent the energy for one unit cell of the lattice. If the lattice is perfectly periodic, then the value of the $n$th term in each sum is clearly independent of $n$. Hence, to evaluate the energy of the infinite 1D lattice, per unit cell, only the $n$th term in these sums needs to be evaluated. If the $n$th term is evaluated for levels $1,2,3$, and so on, these estimates of the total energy should show convergence to a limiting value for the infinite lattice (subject to corrections for nonbonded interactions).

Just as the rules for each fragmentation level can be applied to any arbitrary molecule, so they can be applied to any three-dimensional crystal lattice. The computer algorithm to perform fragmentation of molecules has been described at length previously; thus, only the modifications necessary to produce a fragmentation that respects the periodicity of a crystal lattice are described here.

It is assumed that the lattice vectors $\mathbf{a}(1), \mathbf{a}(2)$, and $\mathbf{a}(3)$ are known, as are the Cartesian coordinates of all $N_{u}$ atoms within a unit cell [the atoms whose fractional coordinates are between 0 and (strictly less than) 1]. These lattice vector and coordinate values may only represent initial estimates and can be optimized, as discussed below. As in the molecular case, two atoms in the crystal are taken to be bonded when their separation in space is less than some value defined by their covalent radii. ${ }^{13,14}$ For example, sphalerite, $\mathrm{ZnS}$, belonging to the cubic space group $F-43 m$, with lattice param-

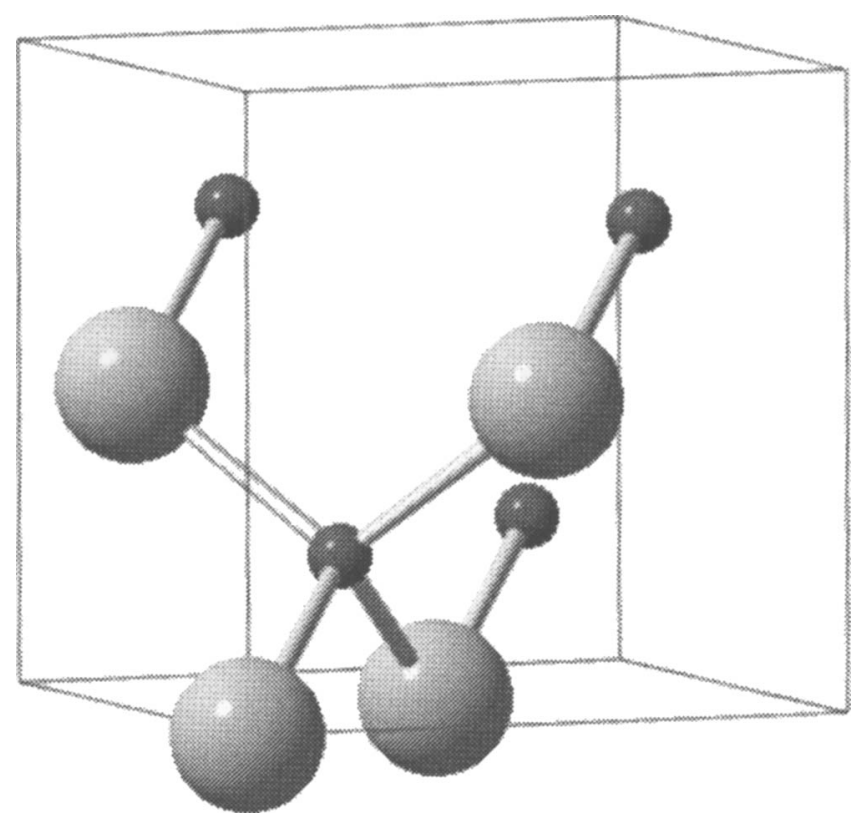

FIG. 1. Schematic representation of the structure of the unit cell of sphalerite (the larger balls denote sulfur atoms; the smaller balls denote zinc atoms).

eters $a=b=c=5.42 \AA$, has eight-atoms in the unit cell, which are bonded together as depicted in Fig. 1. Functional groups of atoms are defined in the same way as for molecules.

The position of any atom in the crystal is given by

$$
\mathbf{x}\left(n, l_{1}, l_{2}, l_{3}\right)=\mathbf{x}(n, 0,0,0)+l_{1} \mathbf{a}(1)+l_{2} \mathbf{a}(2)+l_{3} \mathbf{a}(3),
$$

where $n=1, \ldots, N_{u}\left(N_{u}\right.$ is the number of atoms in the unit cell), and $l_{1}, l_{2}$, and $l_{3}$ are integers. Atoms for which $l_{1}, l_{2}$, and $l_{3}$ are all zero are taken to be the atoms in the "central unit cell." Using Eq. (2.21), the Cartesian coordinates of all atoms are determined, for which $-L \leqslant l_{1}, l_{2}, l_{3} \leqslant L$, for some "cutoff" value of $L$ (typically $L \leqslant 4$ ). This represents a regular block of crystal lattice, as depicted in Fig. 2 for sphalerite.

It can be shown that only fragments containing at least one central unit cell atom are required for a complete description of the fragmentation. Since the fragmentation rules simultaneously break bonds that are separated by some number of groups, two groups cannot be in the same final fragment if they are separated by more than a given number of bonds. This means that atoms in the central unit cell cannot be in fragments with atoms that are separated from them by more than a given number of bonds. For example, Eqs. (2.18)-(2.20) show that the groups $A_{n}, B_{n}$, and $C_{n}$ cannot appear with atoms that are separated from them by more than $k$ bonds at level $k$ fragmentation. In addition, to properly locate the positions of capping hydrogen atoms in the final fragments, the positions of the atoms originally connected to the atoms in all final fragments must be known. Hence, the positions of atoms separated from central unit cell atoms by $k+1$ bonds must be known. So, if a level $k$ fragmentation is specified, the regular block of crystal lattice can be edited to discard all atoms that are more than $k+1$ bonds away from any central unit cell atom. This editing process yields an 


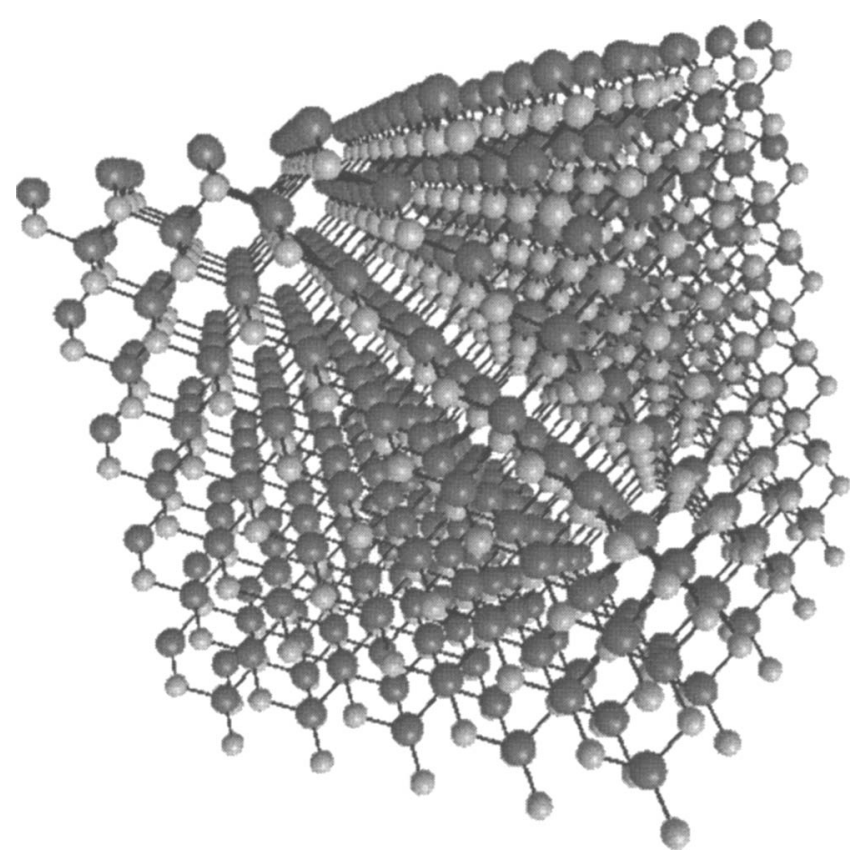

FIG. 2. A segment of 1000 atoms of the sphalerite (ZnS) crystal, representing $5 \times 5 \times 5$ unit cells.

irregular segment of lattice, as illustrated in Fig. 3 for sphalerite. In this example, for level 2 fragmentation, editing reduces the number of atoms to $105, \mathrm{Zn}_{55} \mathrm{~S}_{50}$.

This irregular collection of groups (each group is an atom for sphalerite) can now be fragmented using the same computer algorithm as that used for molecules. ${ }^{14}$ Some modifications of this algorithm have been implemented to improve computational efficiency and to respect the periodicity of the lattice. In summary, as the fragmentation pro-

(a)

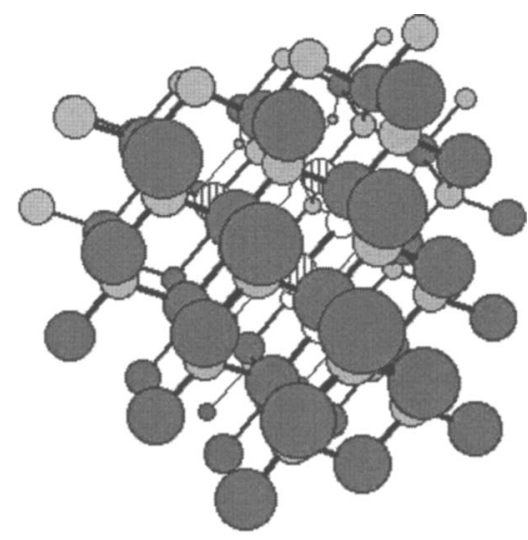

(b)

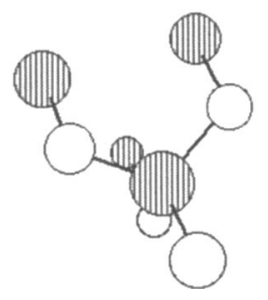

FIG. 3. The subset of $105\left(\mathrm{Zn}_{55} \mathrm{~S}_{50}\right)$ atoms from Fig. 2 that are used to produce a level 2 fragmentation of sphalerite is shown in (a), containing the central unit cell atoms. (b) Zinc atoms in the central unit cell are striped; those in the adjoining cells are dark gray. Sulfur atoms in the central unit cell are white; those in the adjoining cells are light gray. ceeds, a large number of fragments are produced that are themselves further fragmented until the rule at this level can no longer be applied. For crystals, (i) any fragment that does not contain at least one atom from the central unit cell is discarded immediately and (ii) any fragment that is a lattice translation of another fragment is discarded immediately. That is, each atom in one fragment is related to a corresponding atom in another fragment by a lattice translation, and the lattice translation is the same for all atoms.

The "ring repair rule" 14 which precludes fragmentation of sufficiently small rings of connected groups in molecules is also applied to crystal fragmentation. This rule has the effect that four member rings of groups cannot be fragmented at any level, five member rings do not fragment at level 2, six member rings do not fragment at level 3, and so on. This means that some molecules cannot be broken into fragments at some levels of fragmentation. Examples are shown in Ref. 14. In particular, molecules which are composed of fused rings (e.g., decalin) cannot be fragmented at higher levels. This feature prevents the application of systematic fragmentation to crystal structures which are characterized by small fused rings of atoms. So, the diamond structure, in which three six-member rings share an edge, cannot be fragmented at level 3 or above.

By analogy with Eq. (2.14), a final fragment that is associated with the central unit cell is denoted by $F_{n}(0,0,0)$. A fragment that is related by a simple lattice translation is denoted by $F_{n}\left(k_{1}, k_{2}, k_{3}\right)$, and by definition if $\mathbf{x}\left(i, l_{1}, l_{2}, l_{3}\right)$ is contained in $F_{n}(0,0,0)$, then $\mathbf{x}\left(i, k_{1}+l_{1}, k_{2}+l_{2}, k_{3}+l_{3}\right)$ is contained in $F_{n}\left(k_{1}, k_{2}, k_{3}\right)$. Corresponding to Eq. (2.14) for the fragmentation of a molecule, the fragmentation of a crystal lattice can be denoted by

$$
C \rightarrow \sum_{k_{1}=-\infty}^{\infty} \sum_{k_{2}=-\infty}^{\infty} \sum_{k_{3}=-\infty}^{\infty} \sum_{n=1}^{N_{\text {frag }}} c_{n} F_{n}\left(k_{1}, k_{2}, k_{3}\right) .
$$

For the sphalerite example at a level 2, the fragmentation process produces 24 fragments, 4 contain $\mathrm{ZnS}_{4}, 4$ contain $\mathrm{Zn}_{4} \mathrm{~S}$, and 16 contain $\mathrm{ZnS}$, with appropriate hydrogen caps attached. Equation (2.22) is illustrated for sphalerite in Fig. 4.

Corresponding to the final set of crystal fragments, the energy of the crystal per unit cell, due to bonded interactions, can be estimated as

$$
E_{\mathrm{UC}}^{\mathrm{b}}=\sum_{n=1}^{N_{\text {frag }}} c_{n} E_{n}\left[\left\{\mathbf{x}[m(i), \mathbf{I}(i)], i=1, \ldots, N_{a}(n)\right\}\right],
$$

where $N_{a}(n)$ is the number of groups in the $n$th fragment, and $\mathbf{x}$ is the Cartesian vector defined in Eq. (2.21). Equation (2.23) explicitly states that the energy of a crystal fragment is a function of the positions of all the atoms in that fragment. Of course, the energy is independent of the six Cartesian coordinates that specify the position and orientation of the fragment.

The energy of the entire crystal lattice is then given by 


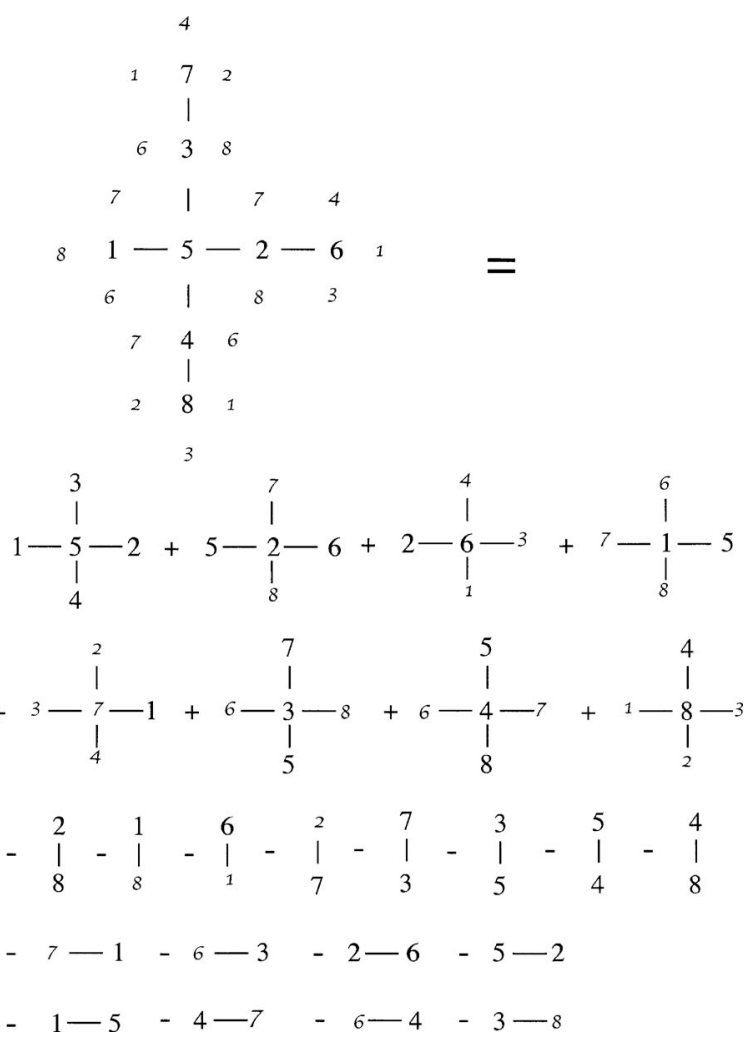

FIG. 4. A schematic, two dimensional representation of the fragmentation of the sphalerite structure at level 2. Atoms 1-4 are sulphur; atoms 5-8 are zinc. The larger font denotes atoms in the central unit cell; the smaller font denotes atoms in adjoining unit cells.

$$
\begin{aligned}
& E_{\text {crys }}^{b}=\sum_{k_{1}=-\infty}^{\infty} \sum_{k_{2}=-\infty}^{\infty} \sum_{k_{3}=-\infty}^{\infty} \sum_{n=1}^{N_{\text {frag }}} c_{n} E_{n}[\{\mathbf{x}[m(i), \mathbf{l}(i)+\mathbf{k}], \\
& i=1, \ldots N_{a}(n)
\end{aligned}
$$

where $\mathbf{k}=\left[k_{1}, k_{2}, k_{3}\right]$ represents a lattice unit cell index. For a perfectly periodic lattice the energy (due to bonding of functional groups) of the crystal per unit cell is given by Eq. (2.23), since all unit cells in Eq. (2.24) have the same energy. The total energy of the crystal per unit cell is given by Eq. (2.23) with the addition of corrections to account for nonbonded interactions.

\section{Nonbonded interactions}

The fragmentation approximation only accounts for the electronic energy associated with functional groups and the bonding between those groups. However, functional groups may be well separated in a crystal in terms of bonding but be close in space, since the crystal structure is threedimensional and the density of atoms is high.

To account for the most important nonbonded interactions, a modified form of many-body expansion is employed. The modification again rests on the assumption that bonded interactions are much stronger than nonbonded interactions.

\section{Two-body interactions}

If $G_{1}$ and $G_{2}$ represent two functional groups in a crystal, then the nonbonded interaction energy between them would be given by

$$
E_{\mathrm{nb}}^{(1,1)}\left[G_{1} ; G_{2}\right]=E\left(G_{1} G_{2}\right)-E\left(G_{1}\right)-E\left(G_{2}\right),
$$

where $E_{\mathrm{nb}}^{(1,1)}\left[G_{1} ; G_{2}\right]$ represents a "two-body" interaction energy between single functional groups, and $E\left(G_{1} G_{2}\right)$ denotes the energy of the two groups, treated as a single molecule. The total energy of the crystal is taken to contain contributions like that in Eq. (2.25) from all possible pairs of functional groups, except where the two groups are contained in any bonded fragment in Eq. (2.22). In the latter case, the interaction energy of these groups has already been accounted for in Eqs. (2.23) and (2.24).

\section{Three-body interactions}

If $G_{1}, G_{2}$, and $G_{3}$ represent three functional groups in a crystal, there is an additional contribution to the crystal energy arising from the fact that the presence of any one group affects the interaction of the other two. That is a three-body effect beyond the sum of the pairwise $E_{\mathrm{nb}}^{(1,1)}$ interactions. It is assumed that this "pure three-body" energy is negligible unless two of the functional groups are directly bonded. That is, the effect of $G_{3}$ on $E_{\mathrm{nb}}^{(1,1)}\left[G_{1}, G_{2}\right]$ is only taken into account if $G_{3}$ is directly bonded to $G_{1}$ or $G_{2}$. Thus, if $G_{3}$ is directly bonded to $G_{2}$,

$$
\begin{aligned}
E_{\mathrm{nb}}^{(1,2)}\left[G_{1} ; G_{2}, G_{3}\right]= & E\left(G_{1} G_{2} G_{3}\right)-E\left(G_{1}\right)-E\left(G_{2} G_{3}\right) \\
& -E_{\mathrm{nb}}^{(1,1)}\left[G_{1}, G_{2}\right]-E_{\mathrm{nb}}^{(1,1)}\left[G_{1}, G_{3}\right] .
\end{aligned}
$$

The total energy of the crystal is taken to contain contributions like that in Eq. (2.26) from all possible combinations of one group, $G_{1}$, with two directly bonded groups $\left(G_{2}, G_{3}\right)$, except where $G_{1}$ appears with either $G_{2}$ or $G_{3}$ in any bonded fragment in Eq. (2.22).

\section{Four-body interactions}

Again, it is assumed that the only significant "pure fourbody" effects are associated with directly bonded groups. There are two types of such four-body interaction energies:

$$
\begin{aligned}
& E_{\mathrm{nb}}^{(2,2)}\left[G_{1} G_{2} ; G_{3} G_{4}\right] \\
&=E\left(G_{1} G_{2} G_{3} G_{4}\right)-E\left(G_{1} G_{2}\right)-E\left(G_{3} G_{4}\right) \\
&-E_{\mathrm{nb}}^{(1,2)}\left[G_{1} ; G_{3}, G_{4}\right]-E_{\mathrm{nb}}^{(1,2)}\left[G_{2} ; G_{3}, G_{4}\right] \\
&-E_{\mathrm{nb}}^{(1,2)}\left[G_{3} ; G_{1} G_{2}\right]-E_{\mathrm{nb}}^{(1,2)}\left[G_{4} ; G_{1} G_{2}\right] \\
&-E_{\mathrm{nb}}^{(1,1)}\left[G_{1} ; G_{3}\right]-E_{\mathrm{nb}}^{(1,1)}\left[G_{1} ; G_{4}\right]-E_{\mathrm{nb}}^{(1,1)}\left[G_{2} ; G_{3}\right] \\
&-E_{\mathrm{nb}}^{(1,1)}\left[G_{2} ; G_{4}\right],
\end{aligned}
$$




$$
\begin{aligned}
E_{\mathrm{nb}}^{(1,3)}\left[G_{1} ; G_{2}, G_{3}, G_{4}\right] \\
=E\left(G_{1} G_{2} G_{3} G_{4}\right)-E\left(G_{1}\right)-E\left(G_{2} G_{3} G_{4}\right) \\
\quad-E_{\mathrm{nb}}^{(1,2)}\left[G_{1} ; G_{2}, G_{3}\right]-E_{\mathrm{nb}}^{(1,2)}\left[G_{1} ; G_{3}, G_{4}\right] \\
\quad-E_{\mathrm{nb}}^{(1,1)}\left[G_{1} ; G_{2}\right]-E_{\mathrm{nb}}^{(1,1)}\left[G_{1} ; G_{3}\right]-E_{\mathrm{nb}}^{(1,1)}\left[G_{1} ; G_{4}\right] .
\end{aligned}
$$

Note that $E_{\mathrm{nb}}^{(2,2)}\left[G_{1} G_{2} ; G_{3}, G_{4}\right]$ vanishes if either $G_{1}$ or $G_{2}$ appears in any bonded fragment with either $G_{3}$ or $G_{4}$ and $E_{\mathrm{nb}}^{(1,3)}\left[G_{1} ; G_{2}, G_{3}, G_{4}\right]$ vanishes if $G_{1}$ appears in any bonded fragment with either $G_{2}, G_{3}$, or $G_{4}$. In $E_{\mathrm{nb}}^{(2,2)}\left[G_{1} G_{2} ; G_{3}, G_{4}\right]$, $G_{1}$ and $G_{2}$ must be directly bonded and $G_{3}$ and $G_{4}$ must be directly bonded, while in $E_{\mathrm{nb}}^{(1,3)}\left[G_{1} ; G_{2}, G_{3}, G_{4}\right], G_{2}$ must be directly bonded to $G_{3}$ which is directly bonded to $G_{4}$.

The expression for the total nonbonded energy for a crystal, including all interactions of up to four groups, is presented in the Appendix. The nonbonded energy per unit cell is easily evaluated from this expression. Calculations for molecules have shown that the analogous five-body effects are sufficiently small to be neglected, and so such higher order effects have not been considered in the calculation of crystal lattice energies.

The best way to evaluate the individual nonbonded interactions is to evaluate each energy in Eqs. (A1)-(A3) by $a b$ initio quantum chemistry. However, this approach would be excessively computationally expensive if all interactions were evaluated in this way. The computational cost is reduced by approximation of the long range nonbonded interactions, as described in the Appendix.

The total energy of the crystal is then given by the sum of $E_{\text {crys }}^{\mathrm{b}}$ in Eq. (2.24) and $E_{\text {crys }}^{\mathrm{nb}}$ in Eq. (A4). The total energy per unit cell is also evaluated by summing $E_{\mathrm{UC}}^{\mathrm{b}}$ in Eq. (2.23) and $E_{\mathrm{UC}}^{\mathrm{nb}}$ in Eq. (A5).

\section{GRADIENTS AND GEOMETRY OPTIMIZATION}

Under periodic conditions, the gradient of the total energy with respect to the position of atom $j$ in unit cell $\mathbf{k}$ is independent of $\mathbf{k}$. Thus, it is sufficient to evaluate the gradient of the potential energy surface with respect to atoms in the UC denoted by $\mathbf{k}=(0,0,0)$.

From Eq. (2.24), the gradient of the bonding energy with respect to the position of the $j$ th atom in the central unit cell is

$$
\begin{aligned}
\frac{\partial E_{\mathrm{crys}}^{\mathrm{b}}}{\partial x_{\alpha}(j, \mathbf{0})}= & \sum_{\mathbf{k}=-\infty}^{\infty} \sum_{n=1}^{N_{\text {frag }}} \sum_{i=1}^{N_{a}(n)} c_{n} \\
& \times \frac{\partial E_{n}\left[\left\{\mathbf{x}[m(i), \mathbf{l}(i)+\mathbf{k}], i=1, \ldots, N_{a}(n)\right\}\right]}{\partial x_{\alpha}[m(i), \mathbf{l}(i)+\mathbf{k}]} \\
& \times \delta_{j, m(i)} \delta_{\mathbf{l}(i)+\mathbf{k}, \mathbf{0}}
\end{aligned}
$$

where $\delta$ denotes the Kronecker delta, and $\delta_{\mathbf{1}(i)+\mathbf{k}, \mathbf{0}}$ should be understood as a product of three Kronecker delta functions, one for each vector component. Since all $\mathbf{k}$ values are included in the sum in Eq. (3.1), the value $\mathbf{k}=-\mathbf{l}(i)$ is present in the sum, so that $\delta_{\mathbf{l}(i)+\mathbf{k}, \mathbf{0}}=1$ for some value of $\mathbf{k}$. This means that there are contributions to the energy gradient $\partial E_{\text {crys }}^{\mathrm{b}} / \partial x_{\alpha}(j, \mathbf{0})$ from every fragment in Eq. (2.23) which contains the $j$ th unit cell atom in some unit cell. Effectively, the energy gradients which are assigned to atoms in the central unit cell are gradients of the bonding energy per unit cell, $E_{\mathrm{UC}}^{\mathrm{b}}$.

The capping hydrogen atoms, contained in each fragment, contribute to the energy gradient for the atoms in the bond which has been broken, since the position of the $\mathrm{H}$ atom is determined completely by the positions of the bonded atoms. The position of each $\mathrm{H}$ atom is taken to lie along the missing bond vector at a distance that is proportional to the expected ratio of bond lengths. That is,

$$
\mathbf{x}(\mathrm{H})=\mathbf{x}(i)+\frac{\operatorname{rad}(i)+\operatorname{rad}(\mathrm{H})}{\operatorname{rad}(i)+\operatorname{rad}(j)}[\mathbf{x}(j)-\mathbf{x}(i)],
$$

where $\mathbf{x}(i)$ denotes the Cartesian position of the atom in the fragment, $\mathbf{x}(j)$ denotes the Cartesian position of the bonded atom not in the fragment, and $\operatorname{rad}(i)$ denotes the covalent radius of atom $i$.

Similarly, the expression for the derivatives of the nonbonded energy, $\partial E_{\text {crys }}^{\mathrm{nb}} / \partial x_{\alpha}(j, \mathbf{0})$, can be obtained by from Eqs. (A1)-(A4). However, since further study is required to determine the most efficient means of evaluating the long range nonbonded interactions, the gradients of the nonbonded energy have not currently been taken into account in geometry optimization.

The energy of the crystal depends on the unit cell parameters (the lattice vectors) because the relative positions of the atoms outside the central unit cell depend on the lattice vectors, as shown in Eq. (2.21). The gradient of the crystal bonding energy per unit cell can be found from Eq. (2.23) from

$$
\begin{aligned}
& \frac{\partial E_{\mathrm{UC}}^{\mathrm{b}}}{\partial a_{\alpha}(v)}=\sum_{i=1}^{n_{\text {atom }}} \frac{\partial E_{\mathrm{UC}}^{\mathrm{b}}}{\partial x_{\alpha}(i)} \frac{\partial x_{\alpha}(i)}{\partial a_{\alpha}}=\sum_{i=1}^{n_{\mathrm{atom}}} \frac{\partial E_{\mathrm{UC}}^{\mathrm{b}}}{\partial x_{\alpha}(i)} l_{v}(i), \\
& \alpha=1,2,3 ; v=1,2,3,
\end{aligned}
$$

where $n_{\text {atom }}$ is the number of atoms which occur in at least one fragment in Eq. (2.23).

Since the unit cell parameters are allowed to change during a geometry optimization, this implies that the volume of the unit cell (and entire crystal) will also change. If the crystal is subject to an isotropic constant external pressure $p$, the Gibbs free energy of the crystal must contain a $p V$ term. The volume of the unit cell can be directly computed from the unit cell parameters

$$
V_{\mathrm{UC}}=\mathbf{a}(1) \cdot[\mathbf{a}(2) \times \mathbf{a}(3)] .
$$

The Gibbs free energy (per unit cell) is then given (in part) as

$$
\Phi_{\mathrm{UC}}=E_{\mathrm{UC}}+p V_{\mathrm{UC}}
$$

Generally, $E_{\mathrm{UC}}$ is the sum of the bonding and nonbonded energies. In the following applications, $E_{\mathrm{UC}}$ is taken to be the bonding energy of Eq. (2.23) only. If we wish to optimize the Gibbs free energy of the crystal with respect to the lattice geometry, then a new term must also be added to the gradient of the crystal energy per unit cell due to this $p V_{\mathrm{UC}}$ term. With a nonzero pressure, Eq. (3.3) is replaced by 


$$
\frac{\partial \Phi_{\mathrm{UC}}}{\partial a_{\alpha}(v)}=\sum_{i=1}^{n_{\text {atom }}} \frac{\partial E_{\mathrm{UC}}}{\partial x_{\alpha}(i)} l_{v}(i)+p \frac{\partial V_{\mathrm{UC}}}{\partial a_{\alpha}(v)} .
$$

So the process for optimization of the crystal structure, based on the UC atoms, begins with a gradient calculation for the fragments in Eq. (2.23) (this is done concurrently with the energy calculation for each fragment). Energy derivatives of the fragments are then assigned to the correct central unit cell atoms, including components due to hydrogen caps. Energy derivatives with respect to the lattice parameters can then be evaluated from Eq. (3.3) and the effect of pressure can be added on as in Eq. (3.6). Herein, geometry optimization has been carried out using the simple steepest descent method. All the Cartesian coordinates of the unit cell atoms and the nine components of the lattice vectors are simultaneously updated at each step in the steepest descent:

$$
\begin{aligned}
& \left.x_{\alpha}(j, \mathbf{0})\right|_{t+\delta t}=\left.x_{\alpha}(j, \mathbf{0})\right|_{t}-\frac{\partial \Phi_{\mathrm{UC}}}{\partial x_{\alpha}(j, \mathbf{0})} \delta t, \\
& \left.a_{\alpha}(v)\right|_{t+\delta t}=\left.a_{\alpha}(v)\right|_{t}-\frac{\partial \Phi_{\mathrm{UC}}}{\partial a_{\alpha}(v)} \delta t
\end{aligned}
$$

where the step size $\delta t$ is adjusted to ensure that the free energy falls monotonically during the optimization. The geometry is taken to be converged when the energy gradients fall below a given tolerance and the change in the Cartesian coordinates, in the last step, is also below a given tolerance.

\section{RESULTS FOR $\mathrm{SiO}_{2}$}

The calculation of lattice energies by the systematic fragmentation approach is illustrated using eight phases of $\mathrm{SiO}_{2}: \beta$-quartz, $\beta$-cristobalite, coesite, keatite, tridymite, $\alpha$-quartz, $\alpha$-cristobalite, and $\alpha$-tridymite. Some properties of these phases are summarized in tables which are available as an EPAPS document. ${ }^{16}$ The structures of most phases of $\mathrm{SiO}_{2}$ have been characterized by diffraction techniques in recent years, but thermochemical data are limited. Quartz is the most stable and common form of silica. At room temperature and standard pressure, it is $\alpha$-quartz. At temperatures above $570{ }^{\circ} \mathrm{C}$, it is stable as the more symmetrical $\beta$-quartz. At $870{ }^{\circ} \mathrm{C}, \beta$-quartz transforms to $\beta$-tridymite and is stable until temperatures of $1470{ }^{\circ} \mathrm{C}$. Tridymite is also stable at temperatures below $870^{\circ} \mathrm{C}$ as $\alpha$-tridymite. At $1470{ }^{\circ} \mathrm{C}, \beta$-tridymite transforms to $\beta$-cristobalite (stable up to temperatures of $1727^{\circ} \mathrm{C}$ ). Cristobalite is metastable between 268 and $1470{ }^{\circ} \mathrm{C}$, but becomes stable again as $\alpha$-cristobalite at temperatures below $268{ }^{\circ} \mathrm{C}$. Cristobalite occurs in igneous rocks in areas of volcanic activity. Coesite is a very rare form of silica, formed under intense heat and pressure (stable above 20 kbars). The few known localities of coesite specimens are meteor crater impact sites. Keatite is a synthetic form of silica (does not exist in nature). It has been produced in the laboratory in the presence of steam over temperatures of $300-600{ }^{\circ} \mathrm{C}$ and pressures between 0.4 and 4 kbars. The structures and data summarized in the EPAPS document were obtained from the CRYSTALMAKER program. ${ }^{17}$

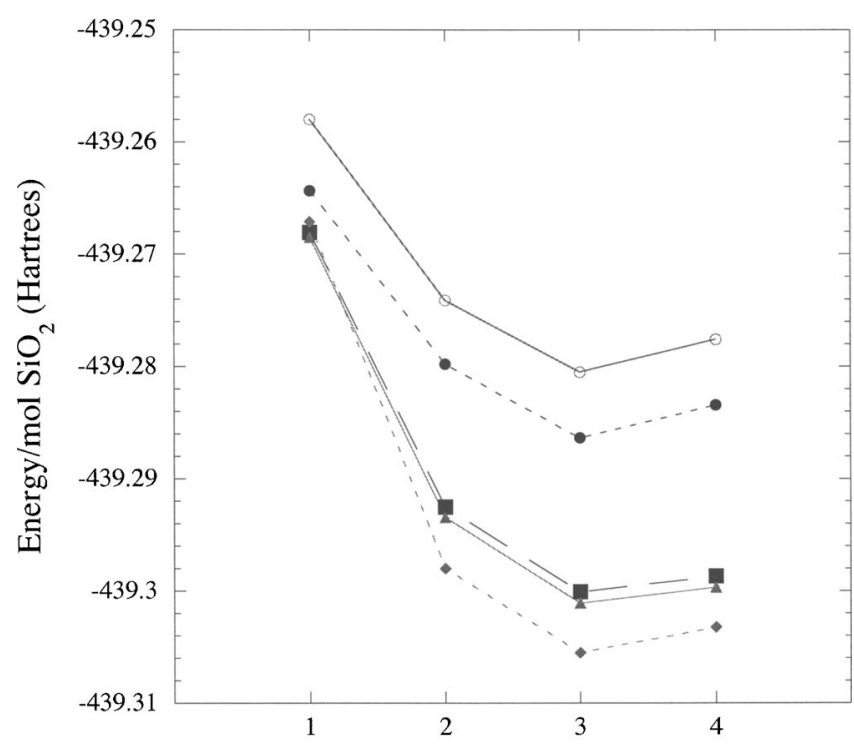

Level of Fragmentation

FIG. 5. The MP2/6-31G $(d, p)$ energy (in hartrees per mole of $\mathrm{SiO}_{2}$ ) is shown, for five polymorphs of $\mathrm{SiO}_{2}$, vs the level of fragmentation: $(\bigcirc)$ tridymite, $(\mathbf{\square})$ keatite, $(\diamond)$ coesite, $(\bullet) \beta$-cristobalite, and $(\mathbf{\Delta}) \beta$-quartz.

The purpose of these illustrations is not to derive the correct structures of these phases at a given temperature and pressure, as that would require consideration of the Gibbs free energy, including entropic contributions from the lattice phonons. The situation is quite complicated for these silicates due to the presence of very low frequency vibrations that affect the SiOSi bond angles. The average silicate structures obtained from diffraction data are simply used here to demonstrate the precision with which lattice energies (and gradients) can be calculated by the fragmentation approach.

\section{A. Fragmentation}

Eight structures for $\mathrm{SiO}_{2}$ have been fragmented using levels 1-4. As an example, keatite, which has $12 \mathrm{Si}$ atoms and $24 \mathrm{O}$ atoms in the unit cell, produces the following pattern of fragments in Eq. (2.22).

Level 1. $48 \mathrm{SiO}-36 \mathrm{Si}-24 \mathrm{O}$.

Level 2. $12 \mathrm{SiO}_{4}+24 \mathrm{Si}_{2} \mathrm{O}-48 \mathrm{SiO}$.

Level 3. $48 \mathrm{Si}_{2} \mathrm{O}_{4}-24 \mathrm{Si}_{2} \mathrm{O}-36 \mathrm{SiO}_{4}$.

Level 4. $12 \mathrm{Si}_{5} \mathrm{O}_{4}+24 \mathrm{Si}_{2} \mathrm{O}_{7}-48 \mathrm{Si}_{2} \mathrm{O}_{4}$.

Capping $\mathrm{H}$ atoms are, of course, added to complete the valence bonding; thus, for example, $\mathrm{O}$ atom fragments are $\mathrm{H}_{2} \mathrm{O}$ molecules, and $\mathrm{Si}_{5} \mathrm{O}_{4}$ fragments are $\mathrm{Si}\left(\mathrm{OSiH}_{3}\right)_{4}$.

\section{B. Energetics}

All $a b$ initio energy and gradient calculations were done with the GAUSSIAN program. ${ }^{18}$ Figures 5 and 6 show the MP2/6-31G $(d, p)$ energy, per mole of $\mathrm{SiO}_{2}$, for each structure (the low temperature $\alpha$ forms are on a separate figure for clarity). Notice that although the structures of these polymorphs are different (see the EPAPS document), the lattice energies per mole of $\mathrm{SiO}_{2}$ are similar in value. The main point to note in Figs. 5 and 6 is that the energy for each structure shows only a small variation, of up to several millihartrees, 


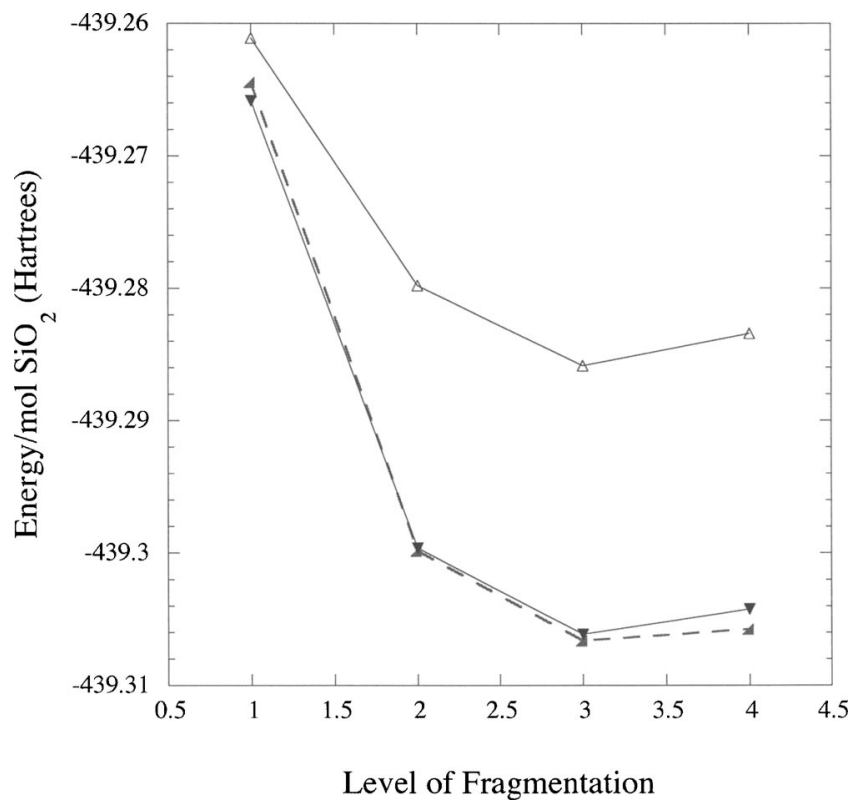

FIG. 6. The MP2/6-31G $(d, p)$ energy (in hartrees per mole of $\mathrm{SiO}_{2}$ ) is shown, for three alpha polymorphs of $\mathrm{SiO}_{2}$, vs the level of fragmentation: ( $\mathbf{C}) \alpha$-quartz, $(\boldsymbol{\nabla}) \alpha$-cristobalite, and $(\triangle) \alpha$-tridymite.

between levels 3 and 4 . The complete energy for these crystal structures must include a smaller nonbonding component, which is not included in Figs. 5 and 6.

Figure 7 shows the effect of including the nonbonded interactions to the level 3 and level 4 bonding energies (using $d_{\text {tol }}=4.5 \AA$, see the Appendix). For these calculations it is clear that the total crystal energy, including nonbonding interactions, is well converged with respect to the level of fragmentation; for $\beta$-cristobalite, $\beta$-quartz, and $\alpha$-quartz, the energy differences between levels 3 and 4 are just 3.7, 2.0, and $2.1 \mathrm{~kJ} \mathrm{~mol}^{-1}$, respectively. These results are consistent with the proposition that the total energy, bonding plus nonbonding, may be converged by levels 3 and 4 , as is the case for organic molecules. ${ }^{14}$

Figure 8 shows the bonding energy of seven polymorphs relative to the energy of tridymite. Again, the relative energies of these phases vary only slowly with fragmentation level by levels 3 and 4 . This indicates that the fragmentation approximation can provide reliable estimates of the relative $a b$ initio energy of these lattices.

Figure 9 presents the energy of the $\beta$-quartz lattice versus level of fragmentation for three different levels of $a b$ initio theory. This graph demonstrates the power of this approach to allow application of high levels of ab initio theory, such as $\operatorname{QCISD}(\mathrm{T})$, to the calculation of lattice energies.

\section{Optimization}

Several of the above structures were optimized (using $\mathrm{HF} / 6-31 \mathrm{G})$ at level 2 fragmentation, using the gradient of the partial free energy, as in Eq. (3.7), at a pressure of $1 \mathrm{~atm}$. The step size in Eq. (3.7), $\delta t$, was initially chosen to be $0.4 \mathrm{bohr}^{2} /$ hartree but was decreased as the energy and coordinates approached convergence. The structures were considered converged when the maximum coordinate and lattice vector changes at a step were below $1.6 \times 10^{-6} \mathrm{bohr}$, and the

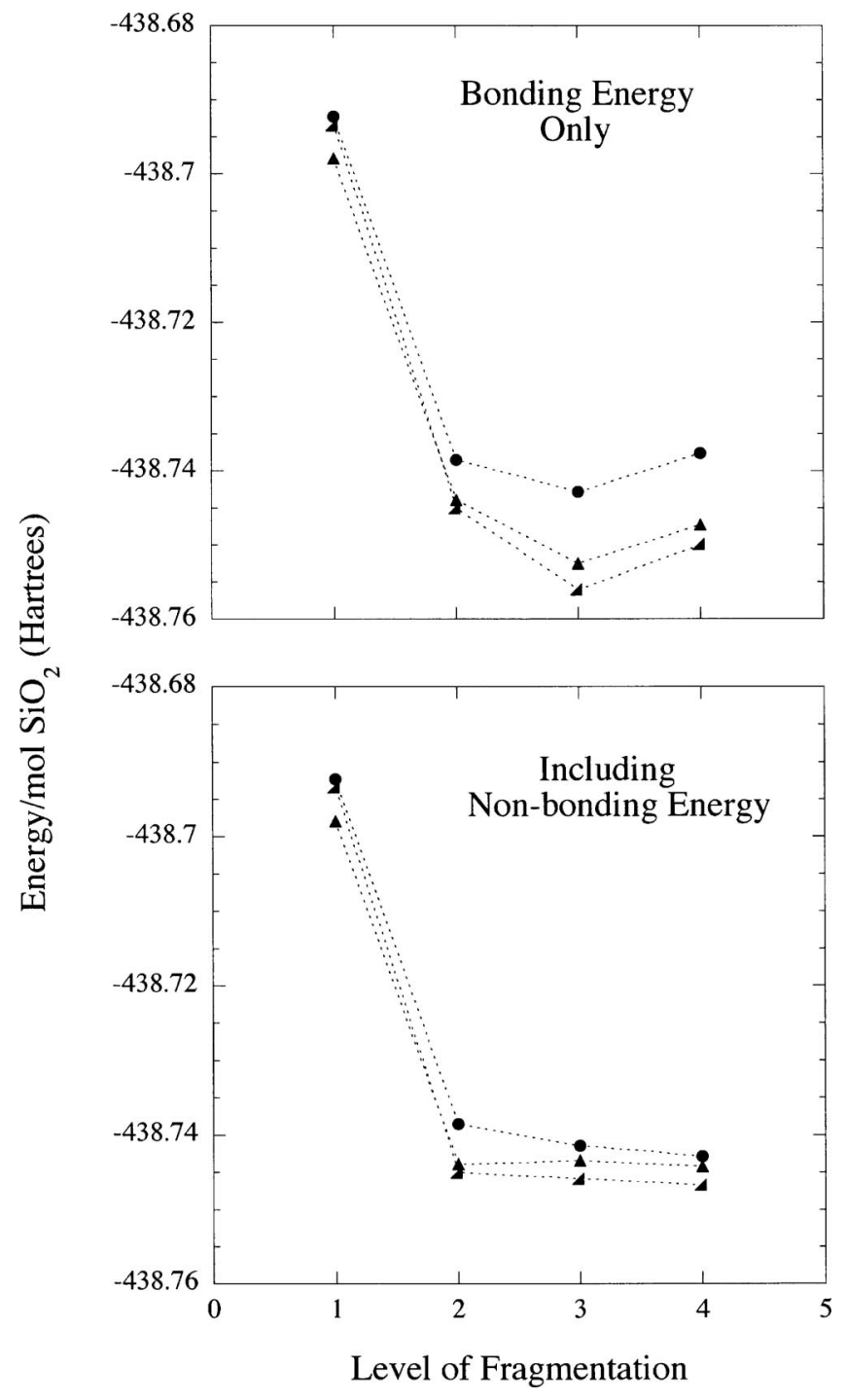

FIG. 7. The HF/6-31G energy (per mole of $\mathrm{SiO}_{2}$ ) is shown, for three polymorphs of $\mathrm{SiO}_{2}$, vs the level of fragmentation in the upper figure and with the addition of the nonbonded energy at levels 3 and 4 in the lower figure:

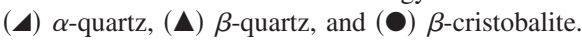

energy change was below $5 \times 10^{-6}$ hartree. To illustrate the results obtained, Table I shows the initial and optimized energies and indicates structural changes for tridymite and $\beta$-quartz.

\section{CONCLUDING REMARKS}

It has been shown, for several crystal structures, that the systematic crystal fragmentation method provides a series of estimates of the lattice energy that converge to within several millihartrees by levels 3 and 4 . With the addition of nonbonded contributions to the energy, lattice energies appear to be converged to within a few $\mathrm{kJ} \mathrm{mol}^{-1}$. A major advantage of the systematic fragmentation method is that lattice energies are estimated by a series of approximations; thus, the error in the best estimate may also be estimated. In principle, if the error is unacceptably large, the fragmentation series may simply be extended to the next level. The second major advantage of the systematic fragmentation approach is that the $a b$ initio calculations required need only be carried out on 


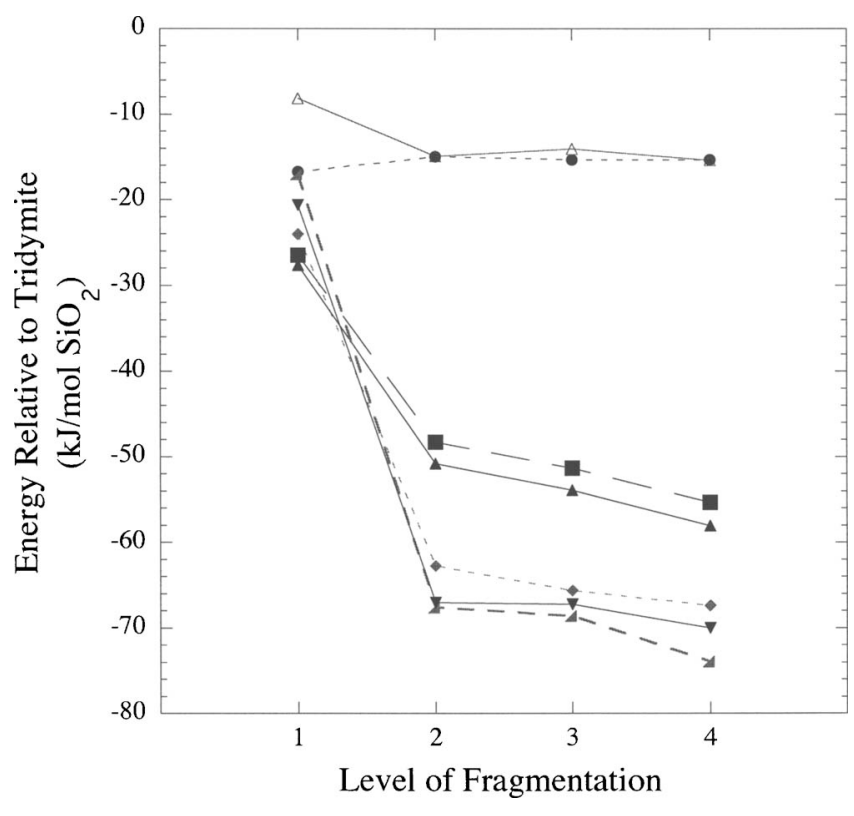

FIG. 8. The MP2/6-31G $(d, p)$ energy (in kJ per mole of $\mathrm{SiO}_{2}$ ) of several $\mathrm{SiO}_{2}$ polymorphs relative to that of tridymite is shown vs the level of frag-

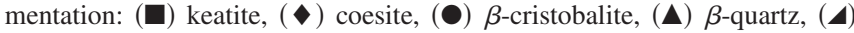
$\alpha$-quartz, $(\mathbf{\nabla}) \alpha$-cristobalite, and $(\triangle) \alpha$-tridymite.

relatively small fragments. This means that the usual "convergence" approach in quantum chemistry can be feasibly employed. The size of the electronic basis set can be increased and the level of treatment of electron correlation can be improved until "converged" estimates of relative energies of crystal structures are obtained. Thus, the usual procedure of establishing convergence of relative energies with respect to basis set and level of $a b$ initio theory is simply augmented with the procedure of establishing convergence with respect to the level of fragmentation.

It is clear that more efficient, accurate methods for esti-

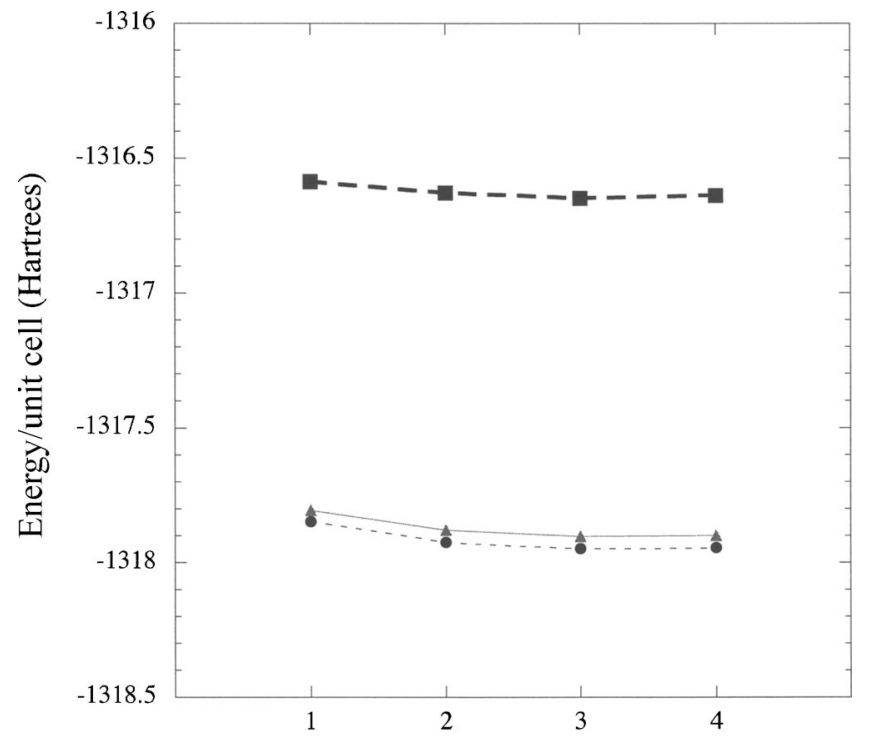

Level of Fragmentation

FIG. 9. The energy of $\beta$-quartz (in hartrees per unit cell) is shown vs the level of fragmentation for three levels of $a b$ initio theory: ( $\boldsymbol{\square})$ HF/6-31G $(d, p),(\mathbf{\Delta}) \mathrm{MP} 2 / 6-31 \mathrm{G}(d, p)$, and (-) QCISD(T)/6-31G $(d, p)$.
TABLE I. The initial and optimized energies per unit cell (hartree), mean Si-O bond lengths $(\AA)$, and mean $\mathrm{Si}-\mathrm{O}-\mathrm{Si}$ bond angles (deg) are presented for level 2 fragmentations of tridymite and $\beta$-quartz.

\begin{tabular}{lcc}
\hline \hline Property & Tridymite & $\beta$-quartz \\
\hline Initial energy & -1754.92719 & -1316.23183 \\
Optimized energy & -1755.05658 & -1316.27424 \\
$\begin{array}{l}\text { Initial mean Si-O } \\
\text { bond length }\end{array}$ & 1.541 & 1.587 \\
$\begin{array}{l}\text { Optimized mean Si-O } \\
\text { bond length }\end{array}$ & 1.633 & 1.640 \\
$\begin{array}{l}\text { Initial mean } \\
\text { Si-O-Si angle }\end{array}$ & 180.0 & 153.4 \\
$\begin{array}{l}\text { Optimized mean } \\
\text { Si-O-Si angle }\end{array}$ & 179.2 & 159.6 \\
\hline \hline
\end{tabular}

mating nonbonding interactions must be included in the calculations. The importance of long range dispersion interactions must be evaluated. Moreover, both bonding and nonbonding interactions must be included in geometry optimization and in calculations of the second derivatives of the lattice energy and the phonon frequencies. This is the minimum information required to optimize the free energy of the lattices with respect to their structure. Properties such as phonon frequencies, the free energy, and phase transitions are the focus of current research, in order to allow meaningful comparison with experimental data.

Obviously, convergence in the calculation of lattice energies has only been demonstrated for several phases of one material. It has not been proved that the systematic fragmentation procedure can be applied universally. In fact, since this type of procedure cannot estimate the energy of organic aromatic ring molecules (e.g., benzene), it is probably safe to assume that the method cannot be applied to any conducting crystals. Moreover, higher levels of the fragmentation procedure cannot be effectively applied to small ring molecules. At these higher levels of fragmentation, the fragments created are simply the whole molecule. Similarly, crystal structures that are characterized by small fused rings of atoms will not be broken up at higher levels of fragmentation. This means that it would be difficult or impossible to demonstrate convergence of estimates of the lattice energy using only fragmentation levels 1 and 2 (say). Hence, there will be many nonconducting materials to which this approach cannot be applied. Nevertheless, structures which are more "open," in the sense that the structure contains larger rings of atoms, should be amenable to study with this approach. Having established the procedures and algorithms reported herein, it will be necessary to investigate a wide range of structure types to clarify the range of applicability of the method.

\section{ACKNOWLEDGMENTS}

The authors would like to acknowledge helpful discussions with Dr. Meredith Jordan and Professor Mark Gordon. One of the authors (H.M.N.) acknowledges funding from the National Science Foundation. Another author (M.A.C.) acknowledges funding from the Australian Research Council. 


\section{APPENDIX: NONBONDING ENERGY}

Let $(n, \mathbf{l})$ denote the $n$th group in the unit cell with lattice integers $\mathbf{l}=\left(l_{1}, l_{2}, l_{3}\right)$. Then $(m, \mathbf{k}) \nsubseteq(n, \mathbf{l})$ denotes that group $(m, \mathbf{k})$ is not contained in any bonded fragment with $(n, \mathbf{l})$, and $(p, \mathbf{j}) \rightarrow(n, \mathbf{l})$ denotes that group $(p, \mathbf{j})$ is directly bonded to group $(n, \mathbf{l})$. Using this notation, the two-, three-, and four-body nonbonded interaction energies for the crystal can be written as

$$
\begin{aligned}
E^{(2)} & =\sum_{\mathbf{l}=-\infty, n=1}^{\infty, N_{u}} \sum_{\substack{\mathbf{k}=-\infty, m=1 \\
(m, \mathbf{k}) \Phi(n, \mathbf{l})}}^{\infty, N_{u}} \frac{1}{2} E_{\mathrm{nb}}^{(1,1)}[(m, \mathbf{k}) ;(n, \mathbf{l})], \\
E^{(3)} & =\sum_{\mathbf{l}=-\infty, n=1}^{\infty, N_{u}} \sum_{\substack{\mathbf{k}=-\infty, m=1 \\
(m, \mathbf{k}) \nsubseteq(n, \mathbf{l})}}^{\infty, N_{u}} \frac{1}{4}\left\{\sum_{\substack{\mathbf{j}=-\infty, p=1 \\
(m, \mathbf{k}) \nsubseteq(p, \mathbf{j}) \\
(p, \mathbf{j}) \rightarrow(n, \mathbf{l})}}^{\infty, N_{u}} E_{\mathrm{nb}}^{(1,2)}[(m, \mathbf{k}) ;(n, \mathbf{l}),(p, \mathbf{j})]+\sum_{\substack{\mathbf{j}=-\infty, p=1 \\
(n, \mathbf{l}) \nsubseteq(p, \mathbf{j}) \\
(p, \mathbf{j}) \rightarrow(m, \mathbf{k})}}^{\infty, N_{u}} E_{\mathrm{nb}}^{(1,2)}[(n, \mathbf{l}) ;(m, \mathbf{k}),(p, \mathbf{j})]\right\},
\end{aligned}
$$

and

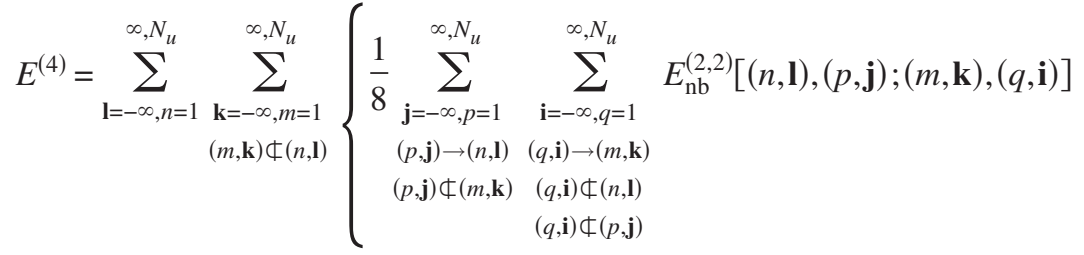

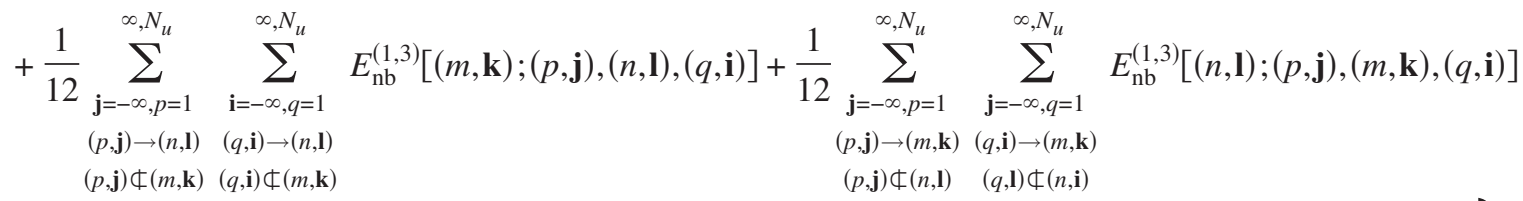

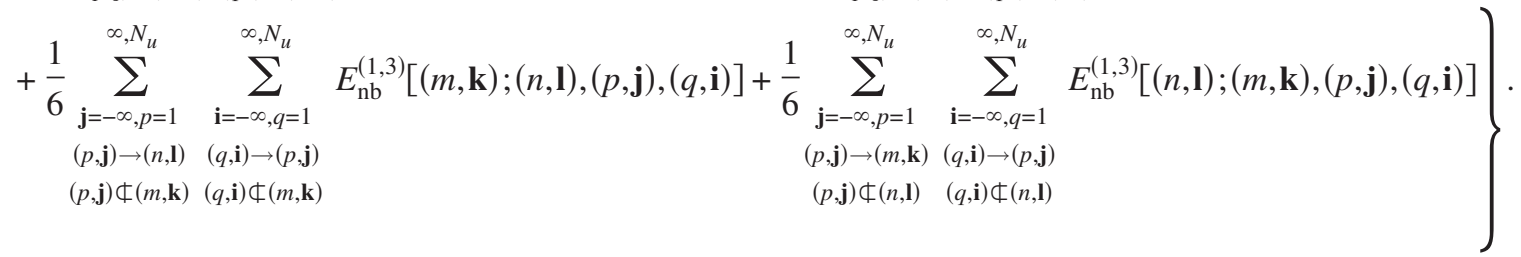

The total nonbonded energy for the crystal is simply estimated as

$$
E_{\text {crys }}^{\mathrm{nb}}=E^{(2)}+E^{(3)}+E^{(4)} .
$$

Setting $\mathbf{l}=(0,0,0)$ in every term in Eq. (A4) gives the nonbonded interaction energy for the whole crystal, per unit cell:

$$
E_{\mathrm{UC}}^{\mathrm{nb}}=\left.E_{\mathrm{crys}}^{\mathrm{nb}}\right|_{\mathrm{l}=(0,0,0)} .
$$

By inspection of Eqs. (A1)-(A3), it is clear that the nonbonded interactions involve a finite number of molecular fragments associated with each unit cell. For a given unit cell [say, $\mathbf{l}=(0,0,0)$ ], these are the $N_{u}$ single groups, the unique pairs of directly bonded groups with at least one member of the pair in the given unit cell, and the directly bonded triple groups, with at least one member of the triple in the given unit cell. The total number of these fragments depends on the crystal structure. For example, for $\beta$-quartz there are 69 such fragments. These fragments can interact with other fragments, except where such interactions have already been accounted for in the bonding energy. Restricting the interactions to involve at most four groups means that the single groups interact with all allowed fragments, the pairs interact only with pairs, and the triples interact only with single groups. These unique fragments are denoted as the "nonbonded bodies."

The total number of interactions contributing to the nonbonded energy per unit cell is only linearly proportional to the number of unit cells that interact with the given unit cell [say, $\mathbf{l}=(0,0,0)]$. Although $E^{(4)}$ in Eq. (A3) appears to involve nested summations over three lattice vectors, $\mathbf{k}, \mathbf{j}$, and $\mathbf{i}$ (for $\mathbf{l}=\mathbf{0}$ ), the requirement that groups be directly bonded reduces this triple sum to a single sum. The remaining infinite sum generally converges rapidly: Terms involving large values of $\|\mathbf{k}\|,\|\mathbf{j}\|$, and $\|\mathbf{i}\|$ are negligible for most crystals. At long range (for example, large values of $\|\mathbf{k}\|,\|\mathbf{j}\|$, and $\|\mathbf{i}\|$ for $\|\mathbf{I}\|=\mathbf{0})$, the interaction between molecular groups can be decomposed into electrostatic, induction, and dispersion effects. ${ }^{19}$ These interactions are inversely proportional to various powers of the distance between the interacting groups, or at very long range, to the distance between the unit cells in which these groups reside. Except for interactions involving charges and dipoles, all these interactions decrease sufficiently rapidly with distance that their sum over distant unit cells converges. Each unit cell in a crystal 


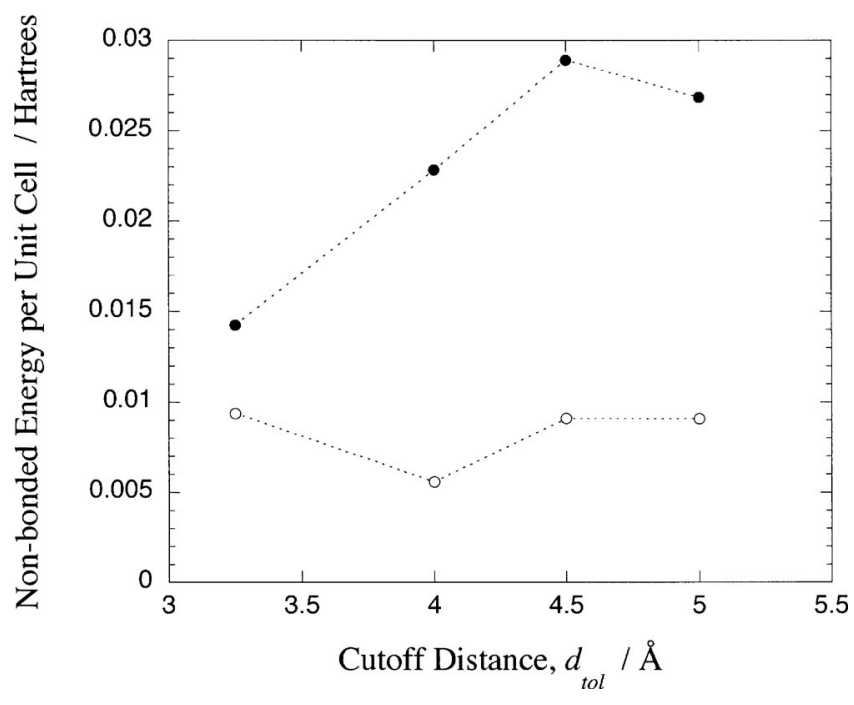

FIG. 10. The total nonbonded energy (ab initio plus long range electrostatic) is shown as a function of the cutoff distance, $d_{\text {tot }}(-)$ level 3 fragmentation and $(\bigcirc)$ level 4 fragmentation.

has no net charge. Only ferroelectric materials have a net dipole moment for a complete unit cell. Hence, with the exception of ferroelectric crystals, Eqs. (A1)-(A3), with $\|\mathbf{I}\|$ $=\mathbf{0}$, can be evaluated by simple summation, truncating the sums when the summand is sufficiently small. That is the simple procedure adopted herein.

The major complication with this simple approach is that the number of interaction energies which must be summed is very large. If all non-negligible interactions were evaluated by $a b$ initio quantum chemical calculations, the resultant computation time would be prohibitive. To remove this problem, we introduce a "cutoff" distance. For any fragmentfragment interaction in Eq. (A4), if the shortest atom-atom distance between the fragments, $d_{\min }$ is less than or equal to some fixed value, $d_{\text {tol }}$, the interaction is evaluated by $a b$ initio calculation. If $d_{\text {min }}$ exceeds $d_{\text {tol }}$, the interaction is evaluated approximately. Herein the long range interactions are estimated by the purely electrostatic interaction of the fragments.

\section{Computational procedure}

$A b$ initio calculations are performed to calculate the energies of the unique nonbonded bodies associated with a unit cell. These energies are required, for example, in Eq. (A1), to calculate the interaction energies between the nonbonded bodies. At the same time, and at no additional cost, the $a b$ initio program $^{18}$ evaluates the dipole, quadrupole, octapole, and hexadecapole moments of each fragment (all fragments are neutral). If $d_{\text {min }}$ exceeds $d_{\text {tol }}$, for some interaction in Eq. (A4), the interaction is estimated from the sum of the electrostatic interactions, as described elsewhere. ${ }^{14}$ The total nonbonded interaction is obtained by combining these energies with $a b$ initio values for the interaction in Eq. (A4) for which $d_{\text {min }}$ does not exceed $d_{\text {tol }}$.

As an example, Fig. 10 presents the resultant total nonbonded interaction energy (per unit cell) for $\beta$-quartz, as a function of the magnitude of $d_{\text {tol }}$ (evaluated using the HF/6$31 \mathrm{G}$ method). Importantly, the figure shows that the total interaction energy becomes insensitive to the value of $d_{\text {tol }}$ when $d_{\text {tol }}$ is $4.5 \AA$ or greater. This suggests that the nonbonded interactions can be accurately calculated while limiting the computational cost of $a b$ initio quantum chemistry calculations. However, two important points must be stressed. Firstly, the data in Fig. 10 refer to Hartree-Fock calculations, in which dispersion effects are absent. In general, it is likely to be necessary to include an accurate approximation for long range dispersion interactions. There are a number of methods to achieve this already in the literature, ${ }^{19,20}$ and current research is directed to devising the most efficient approach. Secondly, a cutoff value as large as $4.5 \AA$ implies that a large number of nonbonded interactions must be evaluated by $a b$ initio calculations. Presumably, at smaller values of $d_{\text {tol }}$ the electrostatic approximation for the interactions of the nonbonded functional groups breaks down because the electrostatic radii of the groups overlap. This problem can probably be ameliorated or even eliminated by using distributed electrostatic moments, as discussed by Stone. ${ }^{19}$ The use of distributed electrostatic moments and polarizabilities is currently under study.

Finally, it is worth noting that the nonbonded interactions between unit cells that are very far apart might be accurately estimated by relatively simple means. In earlier work, it has been noted that the fragmentation approximation for molecular energies can also be applied to the calculation of other molecular properties, including electrostatic moments and polarizabilities. ${ }^{14}$ Hence, the electrostatic moments and polarizabilities of a unit cell can be estimated with increasing accuracy at levels 1-4. Hence, for distant unit cells, the most efficient and accurate estimation of nonbonded interactions may be obtained by calculation of the interaction of whole unit cells rather than by the above many-body approach.

\footnotetext{
${ }^{1}$ E. R. Johnson, R. A. Wolkow, and G. A. DiLabio, Chem. Phys. Lett. 394, 334 (2004).

${ }^{2}$ V. R. Saunders, R. Dovesi, and C. Roetti, CRYSTAL03 User's Manual, Torino, Italy, 2003.

${ }^{3}$ C. Pisani, M. Busso, G. Capecchi, S. Casassa, R. Dovesi, L. Maschio, C. Zicovich-Wilson, and M. Schutz, J. Chem. Phys. 122, 94113 (2005).

${ }^{4}$ P. Y. Ayala and G. E. Scuseria, J. Chem. Phys. 110, 3660 (1999).

${ }^{5}$ G. E. Scuseria and P. Y. Ayala, J. Chem. Phys. 111, 8330 (1999).

${ }^{6}$ P. Y. Ayala, K. N. Kudin, and G. E. Scuseria, J. Chem. Phys. 115, 9698 (2001).

${ }^{7}$ A. Luchow and J. B. Anderson, Annu. Rev. Phys. Chem. 51, 501 (2000).

${ }^{8}$ N. L. Allinger, J. Am. Chem. Soc. 99, 8127 (1977).

${ }^{9}$ N. L. Allinger, Y. H. Yuh, and J. H. Lii, J. Am. Chem. Soc. 111, 8551 (1989).

${ }^{10}$ A. K. Rappe, C. J. Casewit, K. S. Colwell, W. A. Goddard III, and W. M. Skiff, J. Am. Chem. Soc. 114, 10024 (1992).

${ }^{11}$ F. Cortes-Guzman and R. F. W. Bader, Chem. Phys. Lett. 379, 183 (2003).

${ }^{12}$ D. W. Zhang and J. Z. H. Zhang, J. Chem. Phys. 119, 3599 (2003).

${ }^{13}$ V. Deev and M. A. Collins, J. Chem. Phys. 122, 154102 (2005).

${ }^{14}$ M. A. Collins and V. Deev, J. Chem. Phys. 125, 104104 (2006).

${ }^{15}$ S. Humbel, R. D. J. Froese, T. Matsubara, S. Sieber, and K. Morokuma, J. Phys. Chem. 100, 19357 (1996).

${ }^{16}$ See EPAPS Document No. E-JCPSA6-127-318732 for the summary of
} 
crystal structure properties. This document can be reached via a direct link in the online article's HTML reference section or via the EPAPS homepage (http://www.aip.org/pubservs/epaps.html).

${ }^{17}$ D. Palmer and M. Conley, CRYSTALMAKER 7.0.2, CrystalMaker Software Ltd., Oxfordshire, UK, 2005.
${ }^{18}$ M. J. Frisch, G. W. Trucks, H. B. Schlegel et al., GAUSSIAN 03, Gaussian, Inc., Pittsburg, PA, 2003.

${ }^{19}$ A. J. Stone, The Theory of Intermolecular Forces (Clarendon, Oxford, 1996).

${ }^{20}$ I. Adamovic and M. S. Gordon, Mol. Phys. 103, 379 (2005). 